\title{
Incidence and pattern of intraoperative hemodynamic response to endoscopic third ventriculostomy
}

\author{
P. Ganjoo, S. Sethi, M. S. Tandon, R. Chawla, D. Singh ${ }^{1}$ \\ Departments of Anaesthesiology and ${ }^{1}$ Intensive Care and Neurosurgery, GB Pant Hospital, JL Nehru Marg, Nerw Delhi - 110002 , \\ India
}

Address for correspondence:

Dr. Pragati Ganjoo,

Department of Anaesthesiology,

GB Pant Hospital, J L Nehru Marg,

New Delhi - 110 002, India.

E-mail: pganjoo@gmail.com

DOI: $10.4103 / 0028-3886.51285$

\begin{abstract}
Abstrad
Background: In patients undergoing endoscopic third ventriculostomy (ETV), various cardiovascular changes occur in the intraoperative period. Aim: We tried to determine a pattern in these changes and their relation to different surgical steps. Materials and Methods: A total of 260 patients were studied over a period of six years. Heart rate and mean arterial pressures were recorded before introduction of the endoscope and thereafter at various stages of the operation. Results: Tachycardia was the predominant observed abnormality in $20 \%$ of patients, occurring mostly during manipulations and irrigation in the third ventricle (TV). Bradycardia was seen in $12 \%$ of patients, more often during fenestration of the floor of the third ventricle. Conclusions: Tachycardia observed during ETV may be related to hypothalamic stimulation or a rise in intracranial pressure and bradycardia may be due to stimulation of the hypothalamus or the third cranial nerve. Anticipation of these cardiovascular changes during the relevant steps of the operation can help in taking appropriate corrective action, thus preventing potentially serious complications of ETV.
\end{abstract}

Key words: Endoscopic third ventriculostomy, hydrocephalus, neuroendoscopy

\section{Introduction}

Endoscopic third ventriculostomy (ETV) for obstructed hydrocephalus is associated with intraoperative hemodynamic effects ranging from diverse changes in heart rate (HR) and blood pressure (BP) to nearfatal cardiac arrest. ${ }^{[1-7]}$ The possible mechanisms proposed for these changes include inadvertent hypothalamic stimulation or damage,,$^{[1,2,6-8]}$ and acute rise in intracranial pressure (ICP). ${ }^{[4,5,9]}$ However there is yet no clear consensus. Further, different changes have been observed at different stages of ETV, viz. during maneuvering the endoscope within the third ventricle (TV), during ventricular filling with irrigation to improve visibility, or while perforating the TV floor, but it is not clear if the observed changes follow a predictable pattern.

We undertook this prospective observational study to: a) document the incidence of hemodynamic complications during ETV and b) determine the correlation between these changes and different stages of surgery.

\section{M aterials and M ethods}

Consecutive patients undergoing elective ETV by the same neurosurgeon from September 1999 to December 2005 were included in the study. Anesthesia was induced with intravenous (IV) injections of thiopentone $(4 \mathrm{mg} / \mathrm{kg})$, atracurium $(0.5 \mathrm{mg} / \mathrm{kg})$, fentanyl $(2 \mathrm{mcg} / \mathrm{kg})$, and midazolam (1-2 mg). In infants, induction was initiated with halothane before IV access was secured. All patients were intubated and ventilated with an oxygen and nitrous oxide mixture $\left(\mathrm{FiO}_{2} 0.5\right)$ to achieve end-tidal $\mathrm{CO}_{2}\left(\mathrm{EtCO}_{2}\right)$ values of $30-33 \mathrm{~mm} \mathrm{Hg}$. Anesthesia was maintained with atracurium infusion $(10 \mathrm{mcg} / \mathrm{kg} / \mathrm{min})$, $0.5 \%$ isoflurane, and fentanyl boluses. Monitoring included electrocardiogram, pulse oximetry, $\mathrm{EtCO}_{2^{\prime}}$ nasopharyngeal temperature, and invasive arterial blood pressure. Patients were placed supine with head in a 
neutral position on a horseshoe head-rest. Forced-air blankets were used to keep the patients warm. At the end of the procedure, reversal was with IV neostigmine $(0.05 \mathrm{mg} / \mathrm{kg})$ and IV glycopyrrolate $(0.01 \mathrm{mg} / \mathrm{kg})$. Postoperatively, patients were observed in the intensive care unit for 2-4 hours before transfer to the wards.

\section{Surgical technique}

A rigid neuroendoscope (Karl Storz GmBH, Tottingen, Germany) with a $6 \mathrm{~mm}$ sheath and $1.8 \mathrm{~mm}$ telescope was introduced into the lateral ventricles through a standard burr hole. Continuous irrigation with prewarmed Ringer's lactate fluid was started at slow speed $(10 \mathrm{ml} /$ $\mathrm{min}$ ) and was increased if needed to improve visibility during bleeding. Whenever the TV cavity was felt to be overfilled, fluid was let out through the sheath. The procedure was recorded throughout, with images projected onto a video monitor. The endoscope was advanced through the Foramen of Monro (FOM) into the TV cavity. The TV floor was identified by its prominent landmarks and perforation was done at the optimal site with a bipolar cautery. The opening was dilated to $5-6 \mathrm{~mm}$ with a $3 \mathrm{~F}$ Fogarty balloon catheter. Adequacy of ventriculostomy was judged by oscillations of CSF flow through the fenestration. At completion, the neuroendoscope was withdrawn and operative site closed in layers.

\section{Data collection}

Intraoperative hemodynamic variables, $\mathrm{HR}$, and mean arterial pressure (MAP) were continuously monitored throughout the procedure. Recordings were made:

a) Just before insertion of endoscope in the anesthetized stable patient (baseline values).

b) With any change from the baseline values during the various steps of ETV, viz. during movement of the endoscope through the lateral ventricles and FOM, during manipulations inside the TV cavity, during filling of the TV cavity with irrigation, and during fenestration of the TV floor and balloon dilatation of the hole.

c) When remedial action was taken in response to the observed hemodynamic changes.

Bradycardia or tachycardia was defined as a decrease or increase respectively in $\mathrm{HR}$ of $\geq 20 \%$ from baseline values. Hypotension or hypertension (HT) was defined as similar changes in $\mathrm{BP}$ of $\geq 20 \%$ from baseline values. The observed hemodynamic variations were treated initially by retraction of the associated surgical maneuver, and only if they persisted, was drug treatment given. Data were analyzed to document the incidence and nature of hemodynamic changes vis-à-vis the surgical steps of ETV.

\section{Results}

A total of 260 patients were studied. The preoperative data are listed in Table 1 . The procedure was abandoned in 13 patients $(5 \%)$ due to technical reasons. Of the remaining 247 patients, intraoperative tachycardia occurred in 49 patients $(20 \%)$. In 23 patients, the tachycardia was accompanied by HT. Bradycardia was observed in 30 patients $(12 \%)$ out of which only 2 patients had accompanying HT. In all, 25 patients (10\%) developed HT associated either with bradycardia or tachycardia but never manifesting alone. The highest and lowest values of HR observed during the procedures were 194/min and $42 / \mathrm{min}$, respectively, while the highest and lowest MAP were 152 and $57 \mathrm{~mm} \mathrm{Hg}$, respectively. There was no episode of cardiac arrest in our series.

The pattern of hemodynamic changes in relation to various surgical steps are given in Table 2. Baseline HR and MAP values did not change with introduction of the endoscope into the lateral ventricles but when it was inserted into the TV, one patient had hypotension (a narrow FOM necessitating three attempts at insertion), and another had bradycardia (the endoscope inadvertently touched the TV floor). Tachycardia (either isolated, or accompanied with HT) was the predominant response during endoscopic movement and irrigation in the TV cavity. On being alerted, the surgeons stopped the manipulations and irrigation and had let out the irrigating fluid which resulted in prompt reversion of $\mathrm{HR}$ and $\mathrm{BP}$ changes to baseline values. In two patients where we did not respond to the tachycardia in time, bradycardia followed which

\begin{tabular}{lc}
\hline $\begin{array}{l}\text { Table 1: Demographic data of patients undergoing } \\
\text { endoscopic third ventriculostomy }(\mathbf{N}=\mathbf{2 6 0})\end{array}$ \\
\hline Males & \\
Females & 156 \\
Age (years) & 104 \\
$<2$ & \\
$2-5$ & 111 \\
$5-18$ & 74 \\
$>18$ & 54 \\
Diagnosis & 21 \\
Aqueductal stenosis & \\
Tubercular meningitis & 82 \\
Hydrocephalus with tumors & 65 \\
Neonatal hydrocephalus & 38 \\
Postinfective hydrocephalus & 30 \\
Normal pressure hydrocephalus & 24 \\
Shunt infected meningitis & 12 \\
Dandy Walker syndrome & 6 \\
\hline
\end{tabular}

Table 2: Hemodynamic variation vis á vis surgical steps

\begin{tabular}{ll}
\hline Surgical step & Variation \\
\hline Entry into lateral ventricle & No change \\
Navigation via FOM into TV & Hypotension/bradycardia \\
Manipulations and irrigation in TV & Tachycardia \pm hypertension \\
$\begin{array}{l}\text { Fenestration of TV floor and } \\
\text { inflation of Fogarty }\end{array}$ & Bradycardia \pm hypertension \\
\hline
\end{tabular}


then was normalized with egress of the irrigating fluid. The bradycardia (with or without HT) observed in our study most often occurred during perforation of the TV floor or while dilating the hole by balloon inflation of Fogarty catheter. In two patients, simply touching the floor (that appeared thickened) produced bradycardia. Only in one patient was tachycardia observed during fenestration. These changes were also transient and reverted to normal on withdrawal of the endoscope from the TV floor or when the balloon was deflated. None of our patients required any drug treatment for the hemodynamic changes and no one had any postoperative morbidity related to these changes.

\section{Discussion}

We observed increase as well as decrease in HR and MAP during ETV in our study. Tachycardia was the common HR abnormality, occurring in $20 \%$ of patients, while bradycardia was seen in $12 \%$ of patients. In nearly half the number of patients who had tachycardia, there was associated HT. The pattern of hemodynamic responses appeared to vary with the different steps of ETV. Tachycardia was more common during irrigation and endoscopic manipulations in the TV cavity, while bradycardia was the main response during manipulations on or near the TV floor.

Continuous irrigation to improve visibility during neuroendoscopy can generate significant hemodynamic changes possibly due to acute rises in ICP. The classical response to a raised ICP, bradycardia with HT (Cushing reflex), has also been observed during ETV ${ }^{[3]}$ However, van Aken et al., first recognized the significance of tachycardia and $\mathrm{HT}$ as a reflection of raised ICP, attributing this to acute rises in TV pressures by high-speed irrigation or by obstruction to the outflow of fluid..$^{[4]}$ In a recent ETV study where ICP was also measured, it was shown that a tachycardia and HT response (termed an 'atypical' Cushing reflex) correlated well with decrease in cerebral perfusion pressure (CPP) to $<15 \mathrm{mmHg}{ }^{[5]}$ The HT, seen as the initial response to a fall in CPP was considered to be an adaptive mechanism to restore the reduced CPP, and tachycardia occurred only when the increase in ICP was too fast for adaptation, as may happen with high-flow irrigation or outflow obstruction. Tachycardia preceding bradycardia is considered a better indicator of impaired brain perfusion than bradycardia alone and waiting for the latter to happen during irrigation can lead to further rises in ICP with serious cardiovascular and neurological consequences. ${ }^{[5]}$ Post-tachycardial bradycardia occurred in two of our patients which responded to egress of irrigant and did not result in any related postoperative morbidity.

Not all authors agree to a correlation between increases in ICP and generation of cardiovascular changes during ETV. Bradycardia was reported during fenestration of the TV floor in $43 \%$ patients and $41 \%$ patients in two separate studies, ${ }^{[1,2]}$ and in $26.8 \%$ patients during balloon inflation of Fogarty catheter ${ }^{[7]}$ Direct stimulation of the TV floor and the underlying posterior hypothalamus was implicated as the most probable cause of bradycardia. The hypothalamic nuclei surrounding the TV send excitatory or inhibitory influences via descending autonomic pathways to the brainstem which in turn regulates the cardiac function and vasomotor tone in the body. Inadvertent stimulation or damage to the hypothalamus can alter this supranuclear modulation resulting in sympathetic and parasympathetic responses manifesting as increases or decreases in HR and BP.

Our observations on hemodynamic changes during ETV are close to those of van Aken et al., who reported tachycardia in 10 patients during irrigation and endoscopic manipulations in TV cavity and bradycardia in 5 patients during fenestration of the TV floor. ${ }^{[4]}$ In our study, the tachycardia could well be a result of raised ICP or simply due to hypothalamic stimulation after lateral stretching of the TV. However, since HT is more reflective of raised ICP rather than only changes in HR, the tachycardia and HT seen in our patients during irrigation, is most likely due to raised ICP. Inadvertent injury to the hypothalamic nuclei or to the third cranial nerve which lie in close proximity to the TV floor could be responsible for the bradycardia seen by us. Evidence of third nerve injury was seen in the postoperative period in two patients who had bradycardia at the time of fenestration. Inflation of the Fogarty balloon can also produce local pressure changes with signs that mimic raised ICP, ${ }^{[7]}$ and we too observed bradycardia and HT in two of our patients during balloon inflation. Use of saline as an irrigant is also known to produce HT with reflex bradycardia which may be confused with raised $\mathrm{ICP}^{[4]}$ and hence, we used Ringers lactate for irrigation as it does not cause HT and its ionic composition is close to that of cerebrospinal fluid.

We did not witness any cardiac arrest during ETV, but it has been reported earlier. Handler et al., implicated a forceful and rapid irrigation causing distortion of the hypothalamic nuclei either directly or secondary to regional increases in intra-third ventricular pressures for the cardiac arrest and recommended regulating the speed of irrigation to less than $10 \mathrm{ml} / \mathrm{min}$, using it only when needed. ${ }^{[6]}$ Baykan et al., reported asystole in a patient during balloon inflation, supposedly due to local pressures on the underlying hypothalamus. ${ }^{[7]}$ Usually the hemodynamic changes associated with ETV are transient and respond to simple surgical maneuvers. However, the need to use atropine and other resuscitative measures in Baykan's series underscores the seriousness of these changes. 
Meticulous monitoring is a prerequisite for prompt management of ETV related complications. Beat-to-beat monitoring of HR and MAP by an indwelling arterial catheter is strongly recommended. ${ }^{[3-5]}$ Closely following the surgical steps on the video monitor helps anticipate the hemodynamic change specific to that step. Measuring the intraendoscopic pressures can be useful in detecting rises in ICP, though these measurements can be unreliable at times. ${ }^{[3,9]}$ As there is no consensus yet on the safe upper limit of ICP during irrigation, keeping the $\mathrm{CPP}$ above $40 \mathrm{mmHg}$, independent of the ICP, is considered safe. ${ }^{[5]}$ ICP was not measured in our study and we relied on hemodynamic changes alone to alert the surgeons in time.

We have noticed that hemodynamic variations occurred more often in cases where the surgeons encountered difficulties in performing ETV due to a distorted TV or a narrow FOM or a thickened TV floor secondary to infections in the past. A heightened response to endoscopy may have been generated by these oversensitive ventricles, ${ }^{[6]}$ or perhaps, more than the usual force was inadvertently applied to manipulate the endoscope within the small, distorted ventricles. More hemodynamic episodes were seen in the earlier phase of our series and the recent decline in the numbers has probably occurred with an improved surgical technique and a better patient selection.

In conclusion, we found that the hemodynamic responses occurring during ETV at any given time correlate fairly well with the stage of the operation at that point, reflecting the underlying cause. Tachycardia and HT associated well with operative steps of irrigation, suggesting a raised ICP, while bradycardia occurred more often during manipulations on the TV floor, suggesting hypothalamic stimulation. Anticipation of these changes can thus help prevent potentially lifethreatening complications of this operation.

\section{References}

1. El-Dawlatly AA, Murshid W, El-Khwsky F. Endoscopic third ventriculostomy: A study of intracranial pressure vs. haemodynamic changes. Minim Invasive Neurosurg 1999;42:198-200.

2. El-Dawlatly AA, Murshid WR, Elshimy A, Magboul MA, Samarkandi A, Takrouri MS. The incidence of bradycardia during endoscopic third ventriculostomy. Anesth Analg 2000;91:1142-4.

3. Fàbregas N, López A, Valero R, Carrero E, Caral L, Ferrer E. Anesthetic management of surgical neuroendoscopies: Usefulness of monitoring the pressure inside the neuroendoscope. J Neurosurg Anesthesiol 2000;12:21-8.

4. van Aken J, Struys M, Verplancke T, de Baerdemaeker L, Caemaert J, Mortier E. Cardiovascular changes during endoscopic third ventriculostomy. Minim Invasive Neurosurg 2003;46:198-201.

5. Kalmar AF, van Aken J, Caemaert J, Mortier EP, Struys MM. Value of Cushing reflex as warning sign for brain ischaemia during neuroendoscopy. Br J Anaesth 2005;94:791-9.

6. Handler MH, Abbott R, Lee M. Near-fatal complication of endoscopic third ventriculostomy: Case report. Neurosurgery 1994;35:525-8.

7. Baykan N, Isbir O, Gerçek A, Dağçinan A, Özek MM. Ten years of experience with pediatric neuroendoscopic third ventriculostomy. J Neurosurg Anesthesiol 2005;17:33-7.

8. Anandh B, Madhusudan Reddy KR, Mohanty A, Umamaheshwara Rao GS, Chandramouli BA. Intraoperative bradycardia and postoperative hyperkalemia in patients undergoing endoscopic third ventriculostomy. Minim Invasive Neurosurg 2002;45:154-7.

9. Fàbregas N, Valero R, Carrero E, Tercero J, Caral L, Zevala E, et al. Episodic high irrigation pressure during surgical neuroendoscopy may cause intermittent intracranial circulatory insufficiency. J Neurosurg Anesthesiol 2001;13:152-7.

Accepted on 31-03-2009

Source of Support: Nil, Conflict of Interest: None declared.

\section{Author Help: Reference checking facility}

The manuscript system (ww w.journalonw eb.com) allows the authors to check and verify the accuracy and style of references. The tool checks the references with PubM ed as per a predefined style. Authors are encouraged to use this facility, before submitting articles to the journal.

- The style as well as bibliographic elements should be $100 \%$ accurate, to help get the references verified from the system. Even a single spelling error or addition of issue number/month of publication will lead to an error when verifying the reference.

- Example of a correct style

Sheahan P, O'leary G, Lee G, Fitzgibbon J . Cystic cervical metastases: Incidence and diagnosis using fine needle aspiration biopsy. Otolaryngol Head Neck Surg 2002;127:294-8.

- $\quad$ Only the references from journals indexed in PubMed will be checked.

- $\quad$ Enter each reference in new line, without a serial number.

- Add up to a maximum of 15 references at a time.

- If the reference is correct for its bibliographic elements and punctuations, it will be show $n$ as CORRECT and a link to the correct article in PubM ed will be given.

- If any of the bibliographic elements are missing, incorrect or extra (such as issue number), it will be shown as INCORRECT and link to possible articles in PubM ed will be given. 